\title{
Panel Data, Local Cuts, and Orthogeodesic Models
}

by

\author{
Bent Jesper Christensen* \\ School of Economics and Management \\ CAF, CDME, and CLS \\ University of Aarhus \\ Bldg. 350, University Park \\ DK-8000 Aarhus C \\ DENMARK
}

\begin{abstract}
Orthogeodesic models admit marginal local cuts and therefore separate inference on subparameters is asymptotically justified. Doubly-flat orthogeodesic models admit local cuts marginally and conditionally. Two important empirical models for panel data are used to illustrate this property and demonstrate its usefulness. The relation to local ancillarity and local sufficiency is explored. An alternative characterization of local cuts in terms of curvature is given and shown to be intrinsic. Applications to semiparametric estimation are considered.
\end{abstract}




\section{Introduction}

Orthogeodesic models were introduced by Barndorff-Nielsen and Blaesild (1993) as a class of statistical models characterized by purely geometric properties. Christensen and Kiefer (1994) introduced local cuts to allow justification of separate inference - conditionally and marginally - in a wide class of statistical models. In this paper we consider two important classes of panel data models and show that they are in the orthogeodesic (OG) family and that separate inference on subparameters is justified by the theory of local cuts. This property holds generally: OG models admit marginal local cuts.

\section{Panel Data Models}

In many practical applications of formal statistical models, observations are organized in panels with both a time dimension and a cross-sectional dimension. We consider leading cases of economic applications.

\section{Example 2.1. Gaussian Panel}

In this example we consider an empirical model from many areas of applied economic analysis. For each individual $\mathrm{i}, \mathrm{i}=1, \ldots, \mathrm{I}$, we have data $\mathrm{y}_{\mathrm{ij}}$ across a number of time periods $\mathrm{j}=$ $1, \ldots, \mathrm{J}$, and we wish to relate these to observed regressors $\mathrm{z}_{\mathrm{ij}}$. Here we focus on a well-known life-cycle labor supply model. The consumer is assumed to maximize a lifetime utility function in the form $\sum_{\mathrm{t}=0}^{\mathrm{T}} \beta^{\mathrm{t}} \mathrm{u}\left(\mathrm{c}_{\mathrm{t}}, \ell_{\mathrm{t}}\right)$ where $\beta$ is a discount factor, $\mathrm{u}$ is a utility function increasing in its arguments consumption $\mathrm{c}$ and leisure $\ell$. The maximization is subject to the budget constraint that the value of consumption over the life cycle is equal to the value of labor income plus the value of inital assets. The resulting consumption and labor supply functions are in each period 
functions of that period's prices and a time-independent, unobservable variable $\lambda$ (the marginal utility of wealth) that incorporates the effects of initial assets and prices in all other periods.

With suitable further assumptions the model fits in the framework

$$
\mathrm{y}_{\mathrm{ij}}=\alpha_{\mathrm{i}}+\beta \mathrm{z}_{\mathrm{ij}}+\mathrm{u}_{\mathrm{ij}}
$$

where $\mathrm{u}_{\mathrm{ij}}$ is a zero-mean error term. Often, a Gaussian distribution is adopted for the errors. The interest parameter is the slope $\beta$, but unobserved heterogeneity and enters through the coefficients $\alpha_{\mathrm{i}}$. We are interested in broad panels $\mathrm{Y}_{\mathrm{IJ}}=\left\{\mathrm{y}_{\mathrm{ij}}, \mathrm{Z}_{\mathrm{ij}}: \mathrm{i}=1, \ldots, \mathrm{I}, \mathrm{j}=1, \ldots, \mathrm{J}\right\}$, and asymptotics for $\mathrm{I} \rightarrow \infty$, including in the case where $\mathrm{J}$ is fixed, and in this situation there is no hope of estimating $\alpha$ precisely.

\section{Example 2.2 Inverse Gaussian Panel}

In this example we consider an empirical model from financial economics. Our key interest is in asymmetry of information, and this provides a link to banking and macroeconomics (Greenwald et al., 1984, Gertler, 1988). At each time t, a bank has assets $\mathrm{A}_{\mathrm{t}}$ and liabilities $\mathrm{L}_{\mathrm{t}}$, and the net worth (equity) is $\mathrm{N}_{\mathrm{t}}=\mathrm{A}_{\mathrm{t}}-\mathrm{L}_{\mathrm{t}}$. The assets follow the Ito process

$$
\mathrm{dA}_{\mathrm{t}}=\eta \mathrm{dt}+\zeta \mathrm{dW}_{\mathrm{t}}
$$

where $\left\{\mathrm{W}_{\mathrm{t}}\right\}$ is a standard Wiener process. Since the liabilities represent deposits that are fixed in value we specify $L_{t} \equiv L>0$, so that net worth evolves according to $d_{t}=d A_{t}$, initiated at $N_{o}=A_{o}$ $-\mathrm{L}>0$. The bank becomes insolvent at the first time $t$ when assets $\mathrm{A}_{\mathrm{t}}$ drop below liabilities $\mathrm{L}$. In the data, banks are separated into different initial size categories, say $\mathrm{N}_{\mathrm{io}}, \mathrm{i}=1, \ldots, \mathrm{I}$, and quantities are measured in units of initial net worth, e.g. $\mathrm{n}_{\mathrm{it}}=\mathrm{N}_{\mathrm{it}} / \mathrm{N}_{\mathrm{io}}$ and $\sigma_{\mathrm{i}}=\zeta_{\mathrm{i}} / \mathrm{N}_{\mathrm{io}}$. We assume that $\sigma_{i}=\beta z_{i}$, where $z_{i}$ is observed. 
In this setting, $\mathrm{z}_{\mathrm{i}}$ is a measure of the degree of information asymmetry between banks and customers. Since banks have different asset portfolio compositions we have to allow for different ROEs (returns to equity). As we are looking in particular at banks at risk of failing $\operatorname{ROE} \eta_{i} \leq 0$, and upon normalization we consider the parameter $\alpha_{i}=-\eta_{i} / N_{i o} \geq 0$. If we have $J$ banks in size category $i$, the $j$ 'th bank follows

$$
\mathrm{dn}_{\mathrm{ijt}}=\beta \mathrm{z}_{\mathrm{i}} \mathrm{dW} \mathrm{W}_{\mathrm{ijt}}-\alpha_{\mathrm{i}} \mathrm{dt}
$$

with $n_{i j o}=1, j=1, \ldots, J$. Panel data on the times to bank failure are $T_{I J}=\left\{t_{i j}, Z_{i j}: i=1, \ldots, I, j=1, \ldots\right.$, $J\}$ where $t_{i j}$ denotes the first hitting time for the set $\left\{n_{i j t}=0\right\}$.

For fixed $\mathrm{t}>0$, we have so the density at the absorbing barrier $\mathrm{n}_{\mathrm{ijt}}=0$ is

$$
\mathrm{p}\left(\mathrm{n}_{\mathrm{ijt}}=0 ; \alpha_{\mathrm{i}}, \beta\right)=\frac{1}{(2 \pi \mathrm{t})^{1 / 2} \beta \mathrm{z}_{\mathrm{i}}} \mathrm{e}^{-\frac{1}{2 \beta^{2} \mathrm{z}_{\mathrm{i}}^{2} \mathrm{t}}\left(\alpha_{\mathrm{i}} \mathrm{t}-1\right)^{2}},
$$

i.e. the well-known Gaussian, whereas the density of $t_{i j}$ is in the associated inverse Gaussian form

$$
\mathrm{p}\left(\mathrm{t}_{\mathrm{it}}=\mathrm{t} ; \alpha_{\mathrm{i}}, \beta\right)=\frac{1}{\left(2 \pi \mathrm{t}^{3}\right)^{1 / 2} \beta \mathrm{z}_{\mathrm{i}}} \mathrm{e}^{-\frac{1}{2 \beta^{2} \mathrm{z}_{\mathrm{i}}^{2}}\left(\alpha_{\mathrm{i}} \mathrm{t}-1\right)^{2}},
$$

closely resembling (2.4) (see Cox and Oakes (1984, p. 22)) and correspondingly denoted $\mathrm{N}^{-1}\left(\alpha_{\mathrm{i}}, \beta \mathrm{z}_{\mathrm{i}}\right)$

Suppressing terms not depending on parameters, the log likelihood for the panel data $\mathrm{T}_{\mathrm{IJ}}$ is

$$
\ell(\alpha, \beta)=-\mathrm{IJ} \log \beta+\frac{\mathrm{J}}{\beta^{2}} \Sigma_{\mathrm{i}} \frac{\alpha_{\mathrm{i}}}{\mathrm{z}_{\mathrm{i}}^{2}}-\frac{\mathrm{J}}{2 \beta^{2}} \Sigma_{\mathrm{i}} \frac{\overline{\mathrm{u}}_{\mathrm{i}}}{\mathrm{z}_{\mathrm{i}}^{2}}-\frac{\mathrm{J}}{2 \beta^{2}} \Sigma_{\mathrm{i}} \frac{\alpha_{\mathrm{i}}^{2} \overline{\mathrm{t}}_{\mathrm{i}}}{\mathrm{z}_{\mathrm{i}}^{2}}
$$

where $\overline{\mathrm{t}}_{\mathrm{i}}=\Sigma_{\mathrm{j}} \mathrm{t}_{\mathrm{ij}} / \mathrm{J}$ and $\overline{\mathrm{u}}_{\mathrm{i}}=\Sigma_{\mathrm{j}} \mathrm{t}_{\mathrm{ij}}^{-1} / \mathrm{J}$ are the ordinary and inverse harmonic sample means. The score for $\alpha_{i}$ is 


$$
s_{i}=\frac{J}{\beta^{2} z_{i}^{2}}-\frac{J \alpha_{i} \bar{t}_{i}}{\beta^{2} z_{i}^{2}},
$$

so $\hat{\alpha}_{i}=\bar{t}_{i}^{-1}$, yielding the log profile likelihood

$$
\tilde{\ell}(\beta)=-\mathrm{IJ} \log \beta-\frac{\mathrm{J}}{2 \beta^{2}} \Sigma_{\mathrm{i}} \frac{\overline{\mathrm{v}}_{\mathrm{i}}}{\mathrm{z}_{\mathrm{i}}^{2}}
$$

where $\bar{v}_{i}=\bar{u}_{i}-\bar{t}_{i}^{-1}$ is positive since the inverse operation is convex and so by Jensen's inequality the average inverse exceeds the inverse average. The profile score is

$$
\widetilde{\mathrm{s}}(\beta)=-\frac{\mathrm{IJ}}{\beta}+\frac{\mathrm{J}}{\beta^{3}} \Sigma_{\mathrm{i}} \frac{\overline{\mathrm{v}}_{\mathrm{i}}}{\mathrm{z}_{\mathrm{i}}^{2}}
$$

and the profile likelihood equation $\widetilde{\mathrm{s}}(\beta)=0$ produces

$$
\hat{\beta}=\left(\frac{1}{\mathrm{I}} \sum_{i} \frac{\bar{v}_{i}}{z_{i}^{2}}\right)^{1 / 2} .
$$

The problem is that the profile likelihood is not as well-behaved as we would like. There is not even any guarantee of consistency of $\hat{\beta}$ as $\mathrm{I} \rightarrow \infty$. To see this, it is useful to review some results from Barndorff-Nielsen (1988), who considered the model without regressors, i.e. $z_{i} \equiv 1$. First, the full panel $\left\{\mathrm{t}_{\mathrm{ij}}\right\}_{\mathrm{ij}}$ may be reduced by B-sufficiency to $\left\{\left(\bar{t}_{i}, \bar{u}_{i}\right)\right\}_{i}$, and since $\left(\overline{\mathrm{t}}_{\mathrm{i}}, \bar{u}_{i}\right)$ is in one-to-one correspondence with $\left(\bar{t}_{i}, \bar{v}_{i}\right)$, we may equally consider $\left\{\left(\bar{t}_{i}, \bar{v}_{i}\right)\right\}_{i}$. Furthermore, $\overline{\mathrm{t}}_{\mathrm{i}}$ and $\overline{\mathrm{v}}_{\mathrm{i}}$ are independent, $\overline{\mathrm{J}}_{\mathrm{i}} \sim \mathrm{N}^{-1}\left(\alpha_{\mathrm{i}}, \beta \mathrm{z}_{\mathrm{i}}\right)$, and $\mathrm{J} \overline{\mathrm{v}}_{\mathrm{i}} \sim \beta^{2} \mathrm{z}_{\mathrm{i}}^{2} \chi_{\mathrm{J}-1}^{2}$. It follows that $E\left(\bar{v}_{i} / z_{i}^{2}\right)=\beta^{2}(1-1 / J)$, and so $\hat{\beta}$ is biased and inconsistent as $\mathrm{I} \rightarrow \infty$ for $\mathrm{J}$ fixed, in particular $\hat{\beta} \rightarrow \beta(1-1 / \mathrm{J})^{1 / 2}$.

In a given practical situation, if the analysis has been carried to this stage, remedies of the problems arising in situations such as those illustrated in Examples 2.1 and 2.2 obviously exist. However, the key point is that maximum likelihood cannot be pursued without further analysis. 
Our quest is for an inference principle that allows choosing the appropriate objective based on conditions that can be read directly from the likelihood function.

\section{Local Cuts}

In this section we consider separate inference on individual subparameters, with particular reference to proper cuts (Barndorff-Nielsen (1978)) and the local generalization due to Christensen and Kiefer (1994).

Let the model function be $p(x ; \theta)$, and suppose the parameter $\theta \in \Theta$ may be decomposed as $\theta=(\phi, \psi) \in \Phi \times \Psi$ such that at each point $\mathrm{x} \in \mathrm{X}$ we have

$$
\mathrm{p}(\mathrm{x} ; \theta)=\mathrm{p}(\mathrm{x} ; \phi \mid \mathrm{s}) \mathrm{p}(\mathrm{s} ; \psi)
$$

for a suitable statistic s. Then $\mathrm{s}$ is S-sufficient for $\psi$ and S-ancillary for $\phi$ (Barndorff-Nielsen (1978, p. 50)). This restriction is the essential feature of a proper cut. Separate inference on $\phi$ and $\psi$ is indicated, based on the pseudo-likelihoods $\mathrm{p}(\mathrm{x} ; \mid \mathrm{s})$ and $\mathrm{p}(\mathrm{s} ; \cdot)$, respectively.

Inferential separation is crucial in graphical interaction models (Frydenberg (1990)), many normal theory models (Bellhouse (1990)), models that possess nuisance parameters (Kalbfleisch and Sprott (1970)), partial likelihood situations (Cox (1975)), and in numerous other cases, and may sometimes be justified based on other sufficiency criterions, including Msufficiency and G-sufficiency (Barndorff-Nielsen (1978)) and L-sufficiency (Barndorff-Nielsen (1988)).

To motivate the localization procedure, note that in many practical situations separate inference is appropriate as long as the factorization (3.1) is satisfied to sufficient order of approximation. The exact cut condition is usually unnecessarily stringent in applications. 
Examples are given below where the new generalization allows natural inferential separation even when the standard condition is violated.

To define a local cut s, consider shifting $\phi$ in a neighborhood that shrinks with sample size. We are interested in conditions such that the asymptotic consequences of the shift for the marginal distribution $\mathrm{p}(\mathrm{s} ; \theta)$ are less than when instead $\psi$ is shifted. Similarly, the conditional $\mathrm{p}(\mathrm{x} ; \theta \mid \mathrm{s})$ should be less sensitive to $\psi$-shifts than to $\phi$-shifts. Proportional errors in (3.1) correspond to additive errors in the logarithmic representation given as

$$
\begin{gathered}
\log \mathrm{p}\left(\mathrm{x} ; \phi+\mathrm{n}^{-1 / 2} \varepsilon, \psi\right)-\log \mathrm{p}(\mathrm{x} ; \phi, \psi)=\left[\log \mathrm{p}\left(\mathrm{x} ; \phi+\mathrm{n}^{-1 / 2} \varepsilon, \psi \mid \mathrm{s}\right)-\log \mathrm{p}(\mathrm{x} ; \phi, \psi \mid \mathrm{s})\right]+ \\
{\left[\log \mathrm{p}\left(\mathrm{s} ; \phi+\mathrm{n}^{-1 / 2} \varepsilon, \psi\right)-\log \mathrm{p}(\mathrm{s} ; \phi, \psi)\right],}
\end{gathered}
$$

where $\varepsilon \neq 0$ is a vector conformable with $\phi$ and $\mathrm{n}$ is the sample size. Using a vector $\delta \neq 0$

conformable with $\psi$, the consequences of shifting this subparameter are defined by symmetry. Suppose the orders $\mathrm{s}_{\mathrm{c}}(\phi), \mathrm{f}_{\mathrm{c}}(\psi), \mathrm{s}_{\mathrm{m}}(\psi)$ and $\mathrm{f}_{\mathrm{m}}(\phi)$ are such that

$$
\begin{aligned}
& \log \mathrm{p}\left(\mathrm{x} ; \phi+\mathrm{n}^{-1 / 2} \varepsilon, \psi \mid \mathrm{s}\right)-\log \mathrm{p}(\mathrm{x} ; \phi, \psi \mid \mathrm{s})=\mathrm{O}_{\mathrm{p}}\left(\mathrm{n}^{-\mathrm{s}_{\mathrm{c}}(\phi) / 2}\right), \\
& \log \mathrm{p}\left(\mathrm{x} ; \phi, \psi+\mathrm{n}^{-1 / 2} \delta \mid \mathrm{s}\right)-\log \mathrm{p}(\mathrm{x} ; \phi, \psi \mid \mathrm{s})=\mathrm{O}_{\mathrm{p}}\left(\mathrm{n}^{-\mathrm{f}_{\mathrm{c}}(\psi) / 2}\right), \\
& \log \mathrm{p}\left(\mathrm{s} ; \phi, \psi+\mathrm{n}^{-1 / 2} \delta\right)-\log \mathrm{p}(\mathrm{s} ; \phi, \psi)=\mathrm{O}_{\mathrm{p}}\left(\mathrm{n}^{-\mathrm{s}_{\mathrm{m}}(\psi) / 2}\right), \\
& \log \mathrm{p}\left(\mathrm{s} ; \phi+\mathrm{n}^{-1 / 2} \varepsilon, \psi\right)-\log \mathrm{p}(\mathrm{s} ; \phi, \psi)=\mathrm{O}_{\mathrm{p}}\left(\mathrm{n}^{-\mathrm{f}_{\mathrm{m}}(\phi) / 2}\right) .
\end{aligned}
$$

Then the $\varepsilon$-shift in $\phi$ is asymptotically of less consequence for $\mathrm{p}(\mathrm{s} ; \theta)$ than the $\delta$-shift in $\psi$ if and only if $\mathrm{f}_{\mathrm{m}}(\phi)>\mathrm{s}_{\mathrm{m}}(\psi)$. In the same sense, $\mathrm{p}(\mathrm{x} ; \theta \mid \mathrm{s})$ depends less on $\psi$ than on $\phi$ if and only if $\mathrm{f}_{\mathrm{c}}(\psi)>\mathrm{s}_{\mathrm{c}}(\phi)$. Thus, $\mathrm{f}$ indicates the "fast" and $\mathrm{s}$ the "slow" orders, and subscripts $\mathrm{c}$ and $\mathrm{m}$ indicate the conditional and marginal models, respectively.

The two terms in square brackets in (3.2) are of order $\mathrm{s}_{\mathrm{c}}(\phi)$ and $\mathrm{f}_{\mathrm{m}}(\phi)$, respectively, and the total model function dependence on $\phi$ could be largely through $\mathrm{p}(\mathrm{s} ; \theta)$ unless $\mathrm{f}_{\mathrm{m}}(\phi) \geq \mathrm{s}_{\mathrm{c}}(\phi)$. 
Similar considerations on the $\delta$-shift in $\psi$ lead to the requirement $\mathrm{f}_{\mathrm{c}}(\psi) \geq \mathrm{s}_{\mathrm{m}}(\psi)$. In summary, s is a local cut if

$$
\mathrm{f}_{\mathrm{m}}(\phi)>\mathrm{s}_{\mathrm{m}}(\psi), \mathrm{f}_{\mathrm{c}}(\psi)>\mathrm{s}_{\mathrm{c}}(\phi), \mathrm{f}_{\mathrm{m}}(\phi) \geq \mathrm{s}_{\mathrm{c}}(\phi), \mathrm{f}_{\mathrm{c}}(\psi) \geq \mathrm{s}_{\mathrm{m}}(\psi)
$$

(for details and a characterization in terms of approximately separated Edgeworth expansions, see Christensen and Kiefer (1994)). In applications it turns out to be important to have in addition the notion of a marginal local cut, relaxing the second strict inequality to a weak, i.e. a marginal local cut is defined by the requirements

$$
\mathrm{f}_{\mathrm{m}}(\phi)>\mathrm{s}_{\mathrm{m}}(\psi), \mathrm{f}_{\mathrm{c}}(\psi) \geq \mathrm{s}_{\mathrm{c}}(\phi), \mathrm{f}_{\mathrm{m}}(\phi) \geq \mathrm{s}_{\mathrm{c}}(\phi), \mathrm{f}_{\mathrm{c}}(\psi) \geq \mathrm{s}_{\mathrm{m}}(\psi)
$$

In special cases where either $\psi$ or $\phi$ is not present, the concepts of local ancillarity (Cox (1980)) or local sufficiency (McCullagh (1984)) lead to principles for conditional inference on $\phi$, respectively marginal inference on $\psi$, if the relevant differences in asymptotic orders are at least one (for a first order theory) or two (for a second order theory). Similarly, to quantify the nature of the local property in our case, we say that a local cut is of order q if

$$
\mathrm{f}_{\mathrm{m}}(\phi)-\mathrm{s}_{\mathrm{m}}(\psi) \geq \mathrm{q}, \mathrm{f}_{\mathrm{c}}(\psi)-\mathrm{s}_{\mathrm{c}}(\phi) \geq \mathrm{q}
$$

in (3.4), and if

$$
\mathrm{f}_{\mathrm{m}}(\phi)-\mathrm{s}_{\mathrm{m}}(\psi) \geq \mathrm{q}
$$

we have an order q marginal local cut in (3.5). We are now led to the associated strong inference principle that in a model which admits a local cut of order at least one (two for the second order theory), separate inference on $\phi$ and $\psi$ from the conditional respectively the marginal distribution is indicated. This notion is now explored further and illustrated.

\section{Maximum Marginal Likelihood}


In statistical theory, much interest is focussed on the maximum likelihood estimator (MLE) $\hat{\theta}=\arg \max _{\theta} \mathrm{p}(\mathrm{x} ; \theta)$, where $\mathrm{p}(\cdot ;)$ is the model function. With the introduction of the notion of a marginal local cut, we are led to consider an associated strong principle for marginal inference. In particular, suppose that $\theta=(\phi, \psi)$ and that the parameter of interest is $\psi$. If the model admits a marginal local cut s of order one or higher, inference on $\psi$ in the marginal distribution of $\mathrm{s}$ is indicated, and we consider the maximum marginal likelihood estimator (MMLE) $\tilde{\psi}=\arg \max _{\psi} \mathrm{p}(\mathrm{s} ; \phi, \psi)$. In some cases, this depends on $\phi$, but it is not highly critical which value is used for $\phi$, since by the properties of the marginal local cut the dependence asymptotically wears off relatively fast. In other cases, $\tilde{\psi}$ does not depend on $\phi$ at all.

\section{Example 4.1 Marginal Local Cut in Gaussian Panel}

Consider again Example 2.1 and define the time series (group) averages

$\bar{y}_{\mathrm{i}}=\Sigma_{\mathrm{j}} \mathrm{y}_{\mathrm{ij}} / \mathrm{J}, \mathrm{i}=1, \ldots, \mathrm{I}$, and similarly for $\overline{\mathrm{z}}_{\mathrm{i}}$. The model (2.1) in deviations from group means is then

$$
\widetilde{y}_{i j}=\beta \widetilde{z}_{i j}+\widetilde{u}_{i j}
$$

where $\tilde{y}_{\mathrm{ij}}=\mathrm{y}_{\mathrm{ij}}-\overline{\mathrm{y}}_{\mathrm{i}}$, and similary for $\widetilde{\mathrm{z}}_{\mathrm{ij}}$. It is natural to draw inference on $\beta$, the parameter of interest, in this reduced model. Thus, let $\tilde{y}_{i}=\left(\tilde{y}_{i 1}, \ldots, \widetilde{y}_{i J}\right)$, and similarly for $\widetilde{z}_{i}$ and $\tilde{u}_{i}$. If $u_{i}$ are i.i.d. draws from the $J$-dimensional multivariate normal $\mathrm{N}_{\mathrm{J}}\left(0, \sigma^{2} \mathrm{I}_{\mathrm{J}}\right)$ where $\mathrm{I}_{\mathrm{J}}$ is the $\mathrm{J} \times \mathrm{J}$ identity matrix and $\sigma^{2}>0$ then the marginal distribution for $s=\left\{\tilde{y}_{i}\right\}_{i}$ is easily obtained by noting that $\tilde{y}_{i}$ are independent across individuals $i$ and $\tilde{y}_{\mathrm{i}} \sim \mathrm{N}_{\mathrm{J}}\left(\widetilde{\mathrm{z}}_{\mathrm{i}} \beta, \sigma^{2} \mathrm{M}\right)$, where $\mathrm{M}=\mathrm{I}_{\mathrm{J}}-1_{\mathrm{J}} 1_{\mathrm{J}}^{\prime} / \mathrm{J}$. Here, $1_{\mathrm{J}}$ is a J-vector of ones. Thus, if $\sigma^{2}$ does not depend on $\alpha$, the distribution of s does not involve $\alpha$, either. Of course, $\bar{y}_{i} \sim N\left(\alpha_{i}+\bar{z}_{i} \beta, \sigma^{2} / J\right)$, independently across i. In the notation of Section 3 , we would like the interpretation $\phi=\alpha, \psi=\left(\beta, \sigma^{2}\right)$. Indeed, the conditional distribution $\mathrm{p}(\mathrm{x} ; \theta \mid \mathrm{s})$ 
of the panel $x=Y_{\text {IJ }}$ given s may be identified with the marginal distribution of $\bar{y}=\left\{\bar{y}_{i}\right\}_{i}$ since $\bar{y}$ and $\mathrm{s}$ are independent. This distribution depends on both $\phi$ and $\psi$ so $\mathrm{s}$ is not a proper cut. Nonetheless, it may be proved that under wide conditions s is a marginal local cut, and marginal inference on $\psi$ is indicated thus providing a principled basis for a separate inference procedure common in practical applications; further details on this example may be found in Christensen and Kiefer (1994).

\section{Example 4.2 Marginal Local Cut in Inverse Gaussian Panel}

Consider again Example 2.2. In analogy with Example 4.1 above, we wish to draw separate inference on $\psi=\beta$, and to this end consider $\phi=\alpha$ as an infinite-dimensional nuisance parameter. Thus, we specify $s=\bar{v}=\left\{\bar{v}_{i}\right\}_{i}$, and we must verify that $s$ is a marginal local cut. Again, the conditional distribution $\mathrm{p}(\mathrm{x} ; \theta \mid \mathrm{s})$ of the panel $\mathrm{x}=\mathrm{T}_{\mathrm{IJ}}$ may be identified with the marginal distribution of $\overline{\mathrm{t}}=\left\{\overline{\mathrm{t}}_{\mathrm{i}}\right\}_{\mathrm{i}}$ since $\overline{\mathrm{t}}$ and $\overline{\mathrm{v}}$ are independent. This distribution depends on both $\phi$ and $\psi$ so $s$ is not a proper cut. We first derive the orders $f_{c}(\psi)=f_{c}(\beta)$ and $s_{c}(\phi)=s_{c}(\alpha)$ from $\mathrm{p}(\overline{\mathrm{t}} ; \theta \mid \mathrm{s})=\mathrm{p}(\overline{\mathrm{t}} ; \theta), \theta=(\alpha, \beta)($ see $(3.3))$. We have

$$
\log \mathrm{p}(\overline{\mathrm{t}} ; \theta)=-\mathrm{I} \log \beta+\frac{\mathrm{J}}{\beta^{2}} \Sigma_{\mathrm{i}} \frac{\alpha_{\mathrm{i}}}{\mathrm{z}_{\mathrm{i}}^{2}}-\frac{\mathrm{J}}{2 \beta^{2}} \Sigma_{\mathrm{i}} \frac{\overline{\mathrm{t}}_{\mathrm{i}}^{-1}}{\mathrm{z}_{\mathrm{i}}^{2}}-\frac{\mathrm{J}}{2 \beta^{2}} \Sigma_{\mathrm{i}} \frac{\alpha_{\mathrm{i}}^{2} \overline{\mathrm{t}}_{\mathrm{i}}}{\mathrm{z}_{\mathrm{i}}^{2}}
$$

and to calculate $f_{c}(\beta)$ we first note that to appropriate order $\log \left(\beta+n^{-1 / 2} \delta\right)-\log \beta=$ $\log \left(1+n^{-1 / 2} \delta / \beta\right) \approx n^{-1 / 2} \delta / \beta$ and $\left(\beta+n^{-1 / 2} \delta\right)^{-2}-\beta^{-2}=\left[\left(\beta+n^{-1 / 2} \delta\right) \beta\right]^{-2}\left[\beta^{2}-\left(\beta+n^{-1 / 2} \delta\right)^{2}\right] \approx$ $-\beta^{-4}\left[2 n^{-1 / 2} \delta \beta+n^{-1} \delta^{2}\right]$, so that

$$
\begin{gathered}
\log \mathrm{p}\left(\overline{\mathrm{t}} ; \alpha, \beta+\mathrm{n}^{-1 / 2} \delta\right)-\log \mathrm{p}(\overline{\mathrm{t}} ; \alpha, \beta) \approx-\mathrm{I} \frac{\mathrm{n}^{-1 / 2} \delta}{\beta}+ \\
J \frac{2 n^{-1 / 2} \delta \beta+n^{-1} \delta^{2}}{2 \beta^{4}}\left(\Sigma_{i} \frac{\bar{t}_{i}^{-1}-\alpha_{i}}{z_{i}^{2}}+\Sigma_{\mathrm{i}} \alpha_{i} \frac{\alpha_{i} \bar{t}_{i-1}}{z_{i}^{2}}\right) .
\end{gathered}
$$


Since $\bar{t}_{i} \sim N^{-1}\left(\alpha_{i}, \beta z_{i} / J^{1 / 2}\right)$, it is well-known (see e.g. Johnson and Kotz (1970, p. 140)) that $\mathrm{Et}_{\mathrm{i}}^{-1}=\alpha_{\mathrm{i}}+\beta^{2} \mathrm{z}_{\mathrm{i}}^{2} / \mathrm{J}$ and $\mathrm{E} \overline{\mathrm{t}}_{\mathrm{i}}=1 / \alpha_{\mathrm{i}}$. Upon combining the first term on the right hand side with the first term in parenthesis, and ignoring terms of order $\mathrm{n}^{-1}$ and less, (4.3) has been expressed as the sum of I independent zero-mean random variables. The sample size is $n=I J$, and as $\mathrm{I} \rightarrow \infty$ for $\mathrm{J}$ fixed we get easily from a central limit theorem (CLT) that the total expression is $\mathrm{O}_{\mathrm{p}}(1)$. It follows from (3.3b) that $\mathrm{f}_{\mathrm{c}}(\beta)=0$. Similarly, when perturbing $\alpha=\left\{\alpha_{\mathrm{i}}\right\}_{\mathrm{i}}$ by $\varepsilon=\left\{\varepsilon_{\mathrm{i}}\right\}_{\mathrm{i}}$ we have $\left(\alpha_{\mathrm{i}}+\mathrm{n}^{-1 / 2} \varepsilon_{\mathrm{i}}\right)^{2}-\alpha_{\mathrm{i}}^{2}=2 \alpha_{\mathrm{i}} \mathrm{n}^{-1 / 2} \varepsilon_{\mathrm{i}}+\mathrm{n}^{-1} \varepsilon_{\mathrm{i}}^{2}$, so that

$\log \mathrm{p}\left(\overline{\mathrm{t}} ; \alpha+\mathrm{n}^{-1 / 2} \varepsilon, \beta\right)-\log \mathrm{p}(\overline{\mathrm{t}} ; \alpha, \beta)=\frac{\mathrm{Jn}^{-1 / 2}}{\beta^{2}} \Sigma_{\mathrm{i}} \frac{\varepsilon_{\mathrm{i}}}{\mathrm{z}_{\mathrm{i}}^{2}}-\frac{\mathrm{J}}{2 \beta^{2}} \Sigma_{\mathrm{i}} \frac{\overline{\mathrm{t}}_{\mathrm{i}}\left(2 \alpha_{\mathrm{i}} \mathrm{n}^{-1 / 2} \varepsilon_{\mathrm{i}}+\mathrm{n}^{-1} \varepsilon_{\mathrm{i}}^{2}\right)}{\mathrm{z}_{\mathrm{i}}^{2}}$,

to order $n^{-1}$ a sum of I independent zero-mean terms $\left(E \bar{t}_{i}=1 / \alpha_{i}\right)$, and the CLT yields $f_{c}(\alpha)=0$.

Turning next to the candidate marginal local cut, we have $\mathrm{J} \overline{\mathrm{v}}_{\mathrm{i}} / \beta^{2} \mathrm{z}_{\mathrm{i}}^{2} \sim \Gamma((\mathrm{J}-1) / 2,1 / 2)$, the gamma distribution, so that

$$
\log \mathrm{p}(\overline{\mathrm{v}} ; \theta)=-\mathrm{I}(\mathrm{J}-1) \log \beta-\frac{\mathrm{J}}{2 \beta^{2}} \Sigma_{\mathrm{i}} \frac{\overline{\mathrm{v}}_{\mathrm{i}}}{\mathrm{z}_{\mathrm{i}}^{2}}
$$

and obviously $\mathrm{f}_{\mathrm{m}}(\alpha)=\infty$. Much like before we get

$\log \mathrm{p}\left(\overline{\mathrm{v}} ; \alpha, \beta+\mathrm{n}^{-1 / 2} \delta\right)-\log \mathrm{p}(\overline{\mathrm{v}} ; \alpha, \beta) \approx-\mathrm{I}(\mathrm{J}-1) \frac{\mathrm{n}^{-1 / 2} \delta}{\beta}+\mathrm{J} \frac{2 \mathrm{n}^{-1 / 2} \delta \beta+\mathrm{n}^{-1} \delta^{2}}{2 \beta^{4}} \Sigma_{\mathrm{i}} \frac{\overline{\mathrm{v}}_{\mathrm{i}}}{\mathrm{z}_{\mathrm{i}}^{2}}$

and since $E \bar{v}_{i}=(1-1 / J) \beta^{2} z_{i}^{2}$, we have $s_{m}(\beta)=0$ by the CLT. Clearly, by (3.5) $s=\bar{v}$ is a marginal local cut, and inference on $\beta$ in the marginal distribution of $\mathrm{s}$ is indicated.

To obtain the MMLE, differentiation yields the marginal score

$$
\widetilde{\mathrm{s}}_{\mathrm{m}}(\beta)=-\frac{\mathrm{I}(\mathrm{J}-1)}{\beta}+\frac{\mathrm{J}}{\beta^{3}} \Sigma_{\mathrm{i}} \frac{\overline{\mathrm{V}}_{\mathrm{i}}}{\mathrm{z}_{\mathrm{i}}^{2}},
$$

and the marginal likelihood equation $\widetilde{\mathrm{s}}_{\mathrm{m}}(\beta)=0$ produces 


$$
\tilde{\beta}=\left(\frac{\mathrm{J}}{\mathrm{J}-1} \frac{1}{I} \Sigma_{i} \frac{\bar{v}_{i}}{z_{i}^{2}}\right)^{1 / 2}
$$

Clearly, while the MLE is inconsistent, i.e. $\hat{\beta} \rightarrow \beta(1-1 / \mathrm{J})^{1 / 2}<\beta$ (see Section 2), the MMLE corrects this deficiency, i.e. $\mathrm{E}\left(\widetilde{\beta}^{2}\right)=\beta^{2}$ and $\widetilde{\beta} \rightarrow \beta$.

\section{Orthogeodesic Models}

An important new class of parametric statistical models, termed the orthogeodesic family, has recently been introduced by Barndorff-Nielsen and Blaesild (1993). Assuming the statistical model $M=\{p(x, \theta), \theta \in \Theta\}$ is a differentiable manifold, the orthogeodesic property is geometric and may be characterized in general differential geometric terms. The conditions are essentially that (1) $\mathrm{M}$ is a product manifold, $\mathrm{M}=\Phi \times \Psi$, (2) the factorization is orthogonal with respect to the Fisher information metric, (3) when writing $M=\left\{M_{\phi}: \phi \in \Phi\right\}$ the restriction of the metric to $\mathrm{M}_{\phi}$ does not depend on $\phi$, (4) in the sense of Amari (1985), $\mathrm{M}_{\phi}$ is expected $\alpha$-geodesic for some $\alpha \neq 0$, and (5) $\mathrm{M}_{\phi}$ is expected 1-flat.

In parametric terms, an orthogeodesic model (OGM) may be defined by the requirement that there exists a reparametrization $\theta=(\phi, \psi)$ such that this decomposition of $\theta$ corresponds to a geometric factorization as just outlined, and in this case $\theta$ is said to be an ortho-affine parameter. Barndorff-Nielsen and Blaesild (1993) show that with $\phi$ the location and $\sigma$ the scale, the Student $t$ and Cauchy models are orthogeodesic with $\psi$ in the ortho-affine parameter given by $\sigma^{-c}$ and $\log \sigma$, respectively. Here, $\mathrm{c}=2(\mathrm{df}-1) /(\mathrm{df}+5)$, with $\mathrm{df}$ denoting the degrees of freedom. Clearly, many other transformation and exponential models of importance in applications are OGMs. 
Introducing generic coordinates $\mathrm{a}, \mathrm{b}, \mathrm{c} \ldots$ for $\theta ; \mathrm{k}, \ell, \mathrm{m}$ for $\phi$ and $\mathrm{r}, \mathrm{s}, \mathrm{t}$ for $\psi, \mathrm{M}$ can be considered a Riemannian manifold with metric the expected information $\mathrm{i}(\theta)$ (elementwise $\mathrm{i}_{\mathrm{ab}}$; elements of the inverse matrix are $\left.i^{a b}\right)$. The tangent space at $\theta$ is spanned by $\partial_{a} f(\theta)$ for any smooth $\mathrm{f}: \Theta \rightarrow \mathrm{R}$; affine connections $\nabla$ on the associated tangent bundle may be characterized in local coordinates by $\nabla_{\partial \mathrm{a}} \partial_{\mathrm{b}}=\Gamma_{\mathrm{ab}}^{\mathrm{c}} \partial_{\mathrm{c}}$ defining the upper Christoffel symbols, or using the lower Christoffel symbols and the expected information by

$$
\Gamma_{\mathrm{abc}}=\Gamma_{\mathrm{ab}}^{\mathrm{d}} \mathrm{i}_{\mathrm{dc}} .
$$

Of particular interest is the coordinate system defined by the loglikelihood derivatives $\ell_{\mathrm{a}}, \ldots$. The expected 0-connection (the Riemannian connection) is given by

$$
\stackrel{0}{\Gamma}_{\mathrm{abc}}=\frac{1}{2}\left\{\partial_{\mathrm{b}} \mathrm{i}_{\mathrm{ac}}+\partial_{\mathrm{a}} \mathrm{i}_{\mathrm{bc}}-\partial_{\mathrm{c}} \mathrm{i}_{\mathrm{ab}}\right\}
$$

and the corresponding $\alpha$-connections (see Amari 1985) by

$$
\stackrel{\alpha}{\Gamma}_{\mathrm{abc}}=\stackrel{0}{\Gamma} \mathrm{abc}-\frac{\alpha}{2} \mathrm{~T}_{\mathrm{abc}}
$$

with $\mathrm{T}_{\mathrm{abc}}=\mathrm{E}\left(\ell_{\mathrm{a}} \ell_{\mathrm{b}} \ell_{\mathrm{c}}\right)$ the expected skewness. A manifold is $\alpha$-flat if there exists a

parametrization with $\stackrel{\alpha}{\Gamma}_{\mathrm{abc}}=0$. Barndorff-Nielsen and Blaesild (1993) show that conditions 1, 2, and 3 in the characterization of the OGM imply $\stackrel{0}{\Gamma}_{\text {rsk }}=0 ;(1-4)$ imply $\stackrel{\alpha}{\Gamma}_{\text {rsk }}=0$ for all $\alpha$ and $\mathrm{T}_{\text {rsk }}=$ 0 (theorems 4.1 and 4.2). Write

$$
\mathrm{p}(\hat{\phi}, \hat{\psi} \mid \phi, \psi, \mathrm{a})=\mathrm{p}(\hat{\phi} \mid \hat{\psi}, \phi, \psi, \mathrm{a}) \mathrm{p}(\hat{\psi} \mid \phi, \psi, \mathrm{a})
$$

with Edgeworth expansions for each factor

$$
\mathrm{p}(\hat{\phi} \mid \hat{\psi}, \phi, \psi, \mathrm{a}) \approx \mathrm{N}\left(\hat{\phi}, \mathrm{i}_{\phi \phi}^{-1}(\phi, \psi)\right)\left(1+\mathrm{Q}_{\phi \mid \psi}\right)
$$




$$
\mathrm{p}(\hat{\psi} \mid \phi, \psi, \mathrm{a}) \approx \mathrm{N}\left(\psi, \mathrm{i}_{\psi \psi}^{-1}(\psi)\right)\left(1+\mathrm{Q}_{\psi}\right)
$$

where a is ancillary or approximately ancillary and we have used (2) and (3) in specifying the leading normal term. The adjustment terms are

$$
\begin{aligned}
& \mathrm{Q}_{\phi \mid \psi}=\frac{1}{6 \mathrm{n}^{1 / 2}}\left\{\kappa^{\mathrm{abc}} \mathrm{h}_{\mathrm{abc}}\left(\hat{\theta}, \mathrm{i}^{-1}\right)-\kappa^{\mathrm{rst}} \mathrm{h}_{\mathrm{rst}}\left(\hat{\psi}, \mathrm{i}_{\psi \psi}^{-1}\right)\right\} \\
& \mathrm{Q}_{\psi}=\frac{1}{6 \mathrm{n}^{1 / 2}} \kappa^{\mathrm{rst}} \mathrm{h}_{\mathrm{rst}}\left(\hat{\psi}, \mathrm{i}_{\psi \psi}^{-1}\right)
\end{aligned}
$$

The covariant Hermite polynomials $h_{a b c}$ are given in Barndorff-Nielsen and Cox (1989, sec. 5.7), who also show that the indicated approximations are valid to order $\mathrm{n}^{-1}$. We are principally concerned with the coefficients

$$
\kappa^{\mathrm{abc}}=\mathrm{E}\left(\hat{\theta}^{\mathrm{a}}-\theta^{\mathrm{a}}\right)\left(\hat{\theta}^{\mathrm{b}}-\theta^{\mathrm{b}}\right)\left(\hat{\theta}^{\mathrm{c}}-\theta^{\mathrm{c}}\right) .
$$

With this machinery at hand we have

Theorem 1: OG models admit second-order marginal local cuts through $\hat{\psi}$.

Proof: Clearly the leading normal term in the marginal distribution of $\hat{\psi}$ does not depend on $\phi$.

Turning to the coefficients $\kappa^{\text {rst }}$, use the linear relationships $\hat{\psi}^{r}-\psi^{r}=i^{r s} \ell_{s}+O_{p}\left(n^{-1}\right)($ see

Barndorff-Nielsen and Cox (1994)) to write

$$
\kappa^{\mathrm{rst}}=\mathrm{i}^{\mathrm{ru}} \mathrm{i}^{\mathrm{sv}} \mathrm{i}^{\mathrm{tw}} \mathrm{T}_{\mathrm{uvw}}
$$

Condition (5) implies that $\stackrel{1}{\Gamma}_{\text {rst }}=0$ and hence by (5.3) $\mathrm{T}_{\mathrm{uvw}}=\partial_{\mathrm{v}} \mathrm{i}_{\mathrm{uw}}+\partial_{\mathrm{u}} \mathrm{i}_{\mathrm{vw}}-\partial_{\mathrm{w}} \mathrm{i}_{\mathrm{uv}}$, a function only of $\psi$. Since $\phi$ does not appear in $\mathrm{h}_{\mathrm{rst}}\left(\hat{\psi}, \mathrm{i}_{\psi \psi}^{-1}(\psi)\right), \hat{\psi}$ is a second-order $\left(\mathrm{n}^{-1}\right)$ local cut.

Corollary 1. Models satisfying only (1)-(3) admit a (first-order) local cut through $\hat{\psi}$.

Proof: Examine the leading normal term. 
Thus, invoking the strong inference principle from Section 4 , with $s=\hat{\psi}$, separate inference on $\psi$ in OGMs is indicated. With additional conditions, $\hat{\psi}$ becomes a local cut (conditionally as well as marginally). We define the doubly-flat orthogeodesic family as satisfying (1)-(5), (6) $\mathrm{M}_{\psi}$ is geodesic and (7) $\mathrm{M}_{\psi}$ is 1-flat. Then we have

Theorem 2: OGM models satisfying also (6) $\Gamma_{\mathrm{k} \ell}^{\mathrm{\alpha}}=0$ (submanifolds $\mathrm{M}_{\psi}$ are geodesic) and (7) $\Gamma_{l \mathrm{~m}}^{\mathrm{k}}=0\left(\mathrm{M}_{\psi}\right.$ are 1-flat $)$, admit second order local cuts through $\hat{\psi}$.

Proof: Since $\hat{\psi}$ is a second-order marginal local cut by Theorem 1, it remains to be shown that the conditional distribution of $\hat{\phi}$ does not depend on $\psi$ to order $\mathrm{n}^{-1}$. From Barndorff-Nielsen \& Blaesild (1993, Theorem. 4.1) $\stackrel{\alpha}{\Gamma_{\mathrm{k} \ell}^{\mathrm{r}}}=0$ implies $\mathrm{i}_{\mathrm{k} \ell}(\phi, \psi)=\mathrm{i}_{\mathrm{k} \ell}(\phi)$ so the leading normal term does not depend on $\psi$. Turning to the adjustment factor, note that (2) allows elimination of like terms and reduction to

$$
\mathrm{Q}_{\phi \mid \psi}=\frac{1}{6 \mathrm{n}^{1 / 2}}\left\{\kappa^{\mathrm{k} \ell \mathrm{m}} \mathrm{h}_{\mathrm{k} \ell \mathrm{m}}\left(\hat{\phi}, \mathrm{i}_{\phi \phi}^{-1}\right)+3 \kappa^{\mathrm{k} \ell \mathrm{r}} \mathrm{h}_{\mathrm{k} \ell \mathrm{r}}\left(\hat{\theta}, \mathrm{i}^{-1}\right)+3 \kappa^{\mathrm{krs}} \mathrm{h}_{\mathrm{krs}}\left(\hat{\theta}, \mathrm{i}^{-1}\right)\right\}
$$

with

$$
\begin{aligned}
& \kappa^{\mathrm{k} / \mathrm{m}}=\mathrm{i}^{\mathrm{kn}} \mathrm{i}^{\ell \mathrm{o}} \mathrm{i}^{\mathrm{mp}} \mathrm{T}_{\text {nop }} \\
& \kappa^{\mathrm{k} / \mathrm{r}}=\mathrm{i}^{\mathrm{kn}} \mathrm{i}^{\ell \mathrm{o}} \mathrm{i}^{\mathrm{rt}} \mathrm{T}_{\text {not }} \\
& \kappa^{\mathrm{krs}}=\mathrm{i}^{\mathrm{kn}} \mathrm{i}^{\mathrm{rt}} \mathrm{i}^{\mathrm{su}} \mathrm{T}_{\mathrm{ntu}} .
\end{aligned}
$$

Conditions 1-4 imply $\mathrm{T}_{\text {ntu }}$ and hence $\kappa^{\mathrm{krs}}=0$; the additional condition (6) implies $\mathrm{T}_{\text {not }}$ and hence $\kappa^{\mathrm{k} / \mathrm{r}}=0$. Condition (7) implies $\stackrel{1}{\Gamma} \mathrm{k} \mathrm{m}^{2}=0$ and hence $\mathrm{T}_{\mathrm{nop}}=\partial_{\mathrm{o}} \mathrm{i}_{\mathrm{np}}(\phi)+\partial_{\mathrm{n}} \mathrm{i}_{\mathrm{op}}(\theta)-\partial_{\mathrm{p}} \mathrm{i}_{\mathrm{no}}(\phi)$, a function 
only of $\phi$. The coefficients $\mathrm{i}^{\mathrm{kn}}$ etc. refer only to the $\mathrm{i}_{\phi \phi}(\phi)$ block of $\mathrm{i}$ and do not involve $\psi$ either. Hence $\hat{\psi}$ is a second order local cut.

Corollary 2: Models satisfying (1)-(3) and (6) admit a (first-order) local cut through $\hat{\psi}$.

Proof: Examine the leading normal terms.

Examining the proof of Theorem 2 we can obtain the further result that for OG models (without conditions (6) and (7)) the adjustment term implied by the Edgeworth expansion (5.5) in the conditional distribution is linear in $\hat{\psi}$. This is obtained by noting that $\kappa^{\mathrm{krs}}=0$ and hence $\hat{\psi}$ appears only in the polynomials $h_{k(r}$, which contain only linear terms in $\hat{\psi}$.

It may be conjectured from the above that the OGM family generalizes the class of models possessing proper cuts, as do the classes of models that admit local cuts and marginal local cuts. Within the exponential family, this is in fact the case. Barndorff-Nielsen and Blaesild (1983) introduce two subfamilies of the exponential family with $\theta$-parallel or $\tau$-parallel foliations, both of which are OGMs, and the $\theta$-parallel models coincide with the exponential models permitting proper cuts. Of course, $\tau$-parallel models admit second-order marginal local cuts. Finally, the class of doubly-flat OG models admits full local cuts (marginal and conditional), providing a useful insight into the geometry of local cuts. The class of doubly-flat OGMs is a strict generalization of the class of models admitting proper cuts. This can be easily seen by noting that higher order terms that could be added to the Edgeworth expansions (5.5) and (5.6) involve fourth and higher order cross cumulants that are not restricted by our requirements on the second and third order cumulants.

\section{Example 5.1 Gaussian Panel as OGM}

Considering the normal distribution as the limit of Student $t$ distributions as $\mathrm{df} \rightarrow \infty$, 
$\mathrm{N}\left(\phi, \sigma^{2}\right)$ is an OGM with $\psi$ in the ortho-affine parameter given by $\psi=1 / \sigma^{2}$. This is the distribution employed in the Gaussian panel of Examples 2.1, 4.1, and in many empirical applications to life-time labor supply. In fact, other orthogeodesic specifications are useful in economic applications, too, including the Student $t$ with $\mathrm{df}<\infty$ as the distribution of stock returns (which are observed to be more fat-tailed than in the Gaussian case), and panel data models with OGM errors are natural tools for their analysis.

\section{Example 5.2 Inverse Gaussian Panel as OGM}

The inverse Gaussian distribution $\mathrm{N}^{-1}(\alpha, \beta)$ possesses a $\tau$-parallel foliation and so is an

OGM. In this case the ortho-affine parameter is $(\phi, \psi)=\left(\alpha^{-1}, \beta^{-2}\right)$ and even though the scores $\ell_{\phi}$ and $\ell_{\psi}$ are not independent, $\ell_{\phi}$ is independent of the residual from the quadratic regression of $\ell_{\psi}$ on $\ell_{\phi}$ to order $\mathrm{O}_{\mathrm{p}}\left(\mathrm{n}^{-1}\right)$ (Barndorff-Nielsen and Blaesild (1992)). By Theorem 1, the MLE $\hat{\psi}$ of $\psi$ is a second order marginal local cut, and separate inference of $\psi$ in the marginal distribution of $\mathrm{s}$ $=\hat{\psi}$ is indicated. By the invariance of maximum likelihood, $\hat{\psi}=\hat{\beta}^{-2}$, and we may equally consider marginal inference on $\beta$ in the distribution of $\hat{\beta}$. In the inverse Gaussian panel of Examples 2.2 and $4.2 \mathrm{~J}_{\mathrm{i}} /\left(\beta^{2} \mathrm{z}_{\mathrm{i}}^{2}\right) \sim \Gamma((\mathrm{J}-1) / 2,1 / 2)$, so the marginal log likelihood based on $\hat{\beta}$ is

$$
\log \mathrm{p}(\hat{\beta} ; \beta)=-\mathrm{I}(\mathrm{J}-1) \log \beta-\frac{\mathrm{IJ}}{2 \beta^{2}} \hat{\beta}^{2} .
$$

The resulting marginal score is

$$
\widetilde{\mathrm{s}}_{\mathrm{m}}(\beta)=-\frac{\mathrm{I}(\mathrm{J}-1)}{\beta}+\frac{\mathrm{IJ}}{\beta^{3}} \hat{\beta}^{2},
$$

thus producing the MMLE 


$$
\widetilde{\beta}=(1-1 / J)^{-1 / 2} \hat{\beta} .
$$

Thus, the desirable inference procedure from Example 4.2, based on the MMLE from the marginal distribution of $s=\left\{\bar{v}_{i}\right\}_{i}$, again results. This is important since $\beta$ is the risk-shifting parameter in the asymmetric information banking model of Example 2.2. The procedure may in addition be justified based on modified profile likelihood (Barndorff-Nielsen (1988)), but the main point is that it obtains simply by treating the MLE of $\psi$ in the ortho-affine parametrization as a second order marginal local cut.

The analysis reveals important relationships between local cuts and orthogeodesic models. In particular, orthogeodesic models always allow separate inference via the theory of local cuts. Of course, not all models admitting local cuts are OGMs. Further, unlike ortho-affine parametrizations, local cuts are invariant to smooth reparametrizations of the form $(\phi, \psi) \rightarrow$ $(\chi(\phi), \omega(\psi))$. On the other hand, an OGM is characterized by the criterion that an ortho-affine parameter exists, while other parametrizations of course may be of interest, too.

\section{Conclusion}

Separate inference on parameters of OG models is justified on the basis of the theory of local cuts. Our analysis demonstrates the close connection between geometric and inferential aspects of statistical models. The practical relevance of the results is illustrated in two important empirical models for panel data. 


\section{References}

Amari, S.-I. (1985): Differential-Geometric Methods in Statistics. Lecture notes in statistics 28. Heidelberg: Springer-Verlag.

Barndorff-Nielsen, O.E. (1978): Information and Exponential Families in Statistical Theory. Chichester: Wiley.

Barndorff-Nielsen, O.E. (1988): Parametric Statistical Models and Likelihood. New York: Springer.

Barndorff-Nielsen, O.E., and P. Blaesild (1983): "Exponential Models with Affine Dual Foliations" Annals of Statistics, 11, 770-782.

Barndorff-Nielsen, O.E., and P. Blaesild (1992): “A Type of Second Order Asymptotic Independece," JRSSB 54: 897-901.

Barndorff-Nielsen, O.E., and P. Blaesild (1993): "Orthogeodesic Models" Annals of Statistics, 21, 1018-1039.

Barndorff-Nielsen, O.E., and D.R. Cox (1989): Asymptotic Techniques for Use in Statistics. London and New York: Chapman and Hall.

Barndorff-Nielsen, O.E., and D.R. Cox (1994): Inference and Asymptotics. New York: Chapman and Hall.

Bellhouse, D.R. (1990): “On the Equivalence of Marginal and Approximate Conditional Likelihoods for Correlation Parameters Under a Normal Model” Biometrika, 77, 743746.

Christensen, B.J., and N.M. Kiefer (1994): "Local Cuts and Separate Inference" Scandinavian Journal of Statistics, 21, 389-407.

Cox, D.R. (1975): "Partial Likelihood” Biometrika, 62, 269-276.

Cox, D.R. (1980): “Local Ancillarity” Biometrika, 67, 279-286.

Cox, D.R., and D. Oakes (1984): Analysis of Survival Data. New York: Chapman and Hall.

Frydenberg, M. (1990): "Marginalization and Collapsibility in Graphical Interaction Models" Annuals of Statistics, 18, 790-805.

Gertler, M. (1988): "Financial Structure and Aggregate Economic Activity: An Overview" Journal of Money, Credit and Banking, 20, 559-588. 
Greenwald, B., Stiglitz, J.E., and A. Weiss (1984): "Informational Imperfections in the Capital Market and Macroeconomic Flutucations" American Economic Review, 74, 194-199.

Johnson, N.L., and S. Kotz (1970): Continuous Univariate Distributions. New York: Wiley.

Kalbfleisch, J.D., and D.A. Sprott (1970): “Applications of Likelihood Methods to Models Involving Large Numbers of Parameters" Journal of the Royal Statistical Society Series $B, 32,175-208$.

McCullagh, P. (1984): “Local Sufficiency” Biometrika, 71, 233-244. 